\title{
APLICABILIDAD DE LA MEDIDA DE LA VELOCIDAD DE CORROSIÓN DE LAS ARMADURAS EN LA AUSCULTACIÓN DE FORJADOS CONSTRUIDOS CON CEMENTO ALUMINOSO
}

\author{
(APPLICABILITY OF REBAR CORROSION RATE MEASUREMENTS TO THE \\ ASSESSMENT OF HIGH ALUMINA CEMENT FLOORS)
}

Luis M. Ortega Basagoiti, Ing. C. C. y P.

Marta García Guillot, Lda. Ciencias Químicas

Jesús Rodríguez Santiago, Dr. Ing. C. C. y P. GEOCISA - ESPAÑA

Fecha de recepción: 11-XI-92 615-10

\section{RESUMEN}

Dentro de los problemas generales de durabilidad que plantean las estructuras de hormigón armado, el hormigón fabricado con cemento aluminoso es un caso muy particular que presenta determinados problemas característicos, al tiempo que padece otros comunes al hormigón de cemento portland, aunque si cabe con perfiles de mayor gravedad.

Estas peculiares características deben ser tenidas en cuenta al abordar campañas de auscultación específicas de los edificios en cuya construcción fue presumiblemente utilizado el cemento aluminoso.

Este artículo tiene por objeto comentar las líneas generales de tal tipo de auscultaciones, presentando las posibilidades de aplicación al caso, de la técnica específica de la medida de la intensidad de corrosión de las armaduras, así como algunos resultados

estadísticos obtenidos a partir de reconocimientos efectuados por el Laboratorio GEOCISA.

\section{SUMMARY}

High alumina cement floors constitute a especial case within the great field of durability problems of $R C$ structures. They present some characteristic problems, and share, even with greater intensity, some others which are common to Portland cement $R C$ structures.

These special characteristics should be taken into account in the assessment tasks specifically intended for buildings presumably constructed using high alumina cement.

This paper describes the general guidelines of those assessments, presenting the applicability and capabilities for these cases of the measurement of rebar corrosion rate. Some statistical data obtained from building revisions developed by GEOCISA are also given.

\section{CONSIDERACIONES PREVIAS}

En los últimos años se ha ido poco a poco modificando la idea errónea, pero muy extendida hace algún tiempo, de la perdurabilidad casi "eterna" de las estructuras de hormigón armado. La realidad del día a día ha provocado un continuo enfrentamiento con la existencia de muy diferentes problemas en ese material que se creía inal- terable, bien provocados por errores en el propio proyecto, bien por defectos de la ejecución o bien por degradaciones y daños inducidos por acciones externas. El hormigón fabricado con cemento aluminoso es un caso muy particular que presenta determinados problemas característicos, al tiempo que padece otros comunes al hormigón de cemento portland aunque, si cabe, con perfiles de mayor gravedad. 


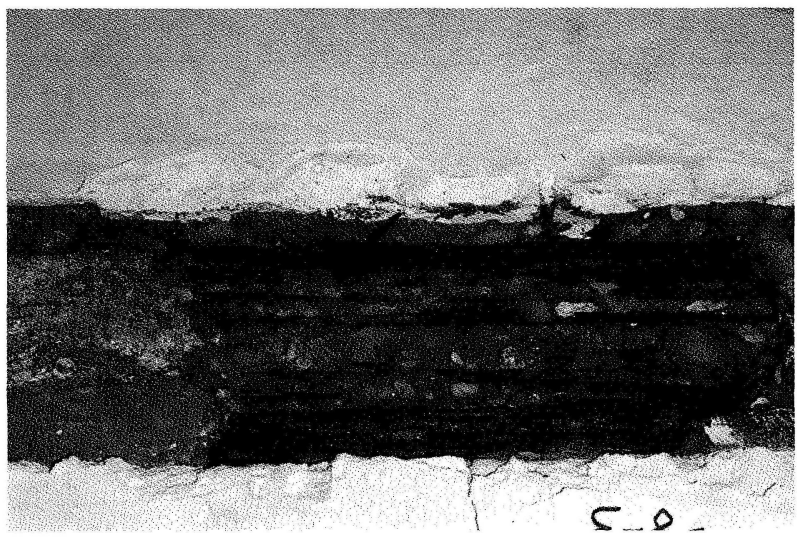

Fig. 1.-Corrosión en las armaduras de una vigueta fabricada con cemento aluminoso.

El empleo en tiempos pasados del cemento aluminoso en la fabricación de viguetas para forjados, sin la observancia estricta de determinadas normas de buena práctica imprescindibles en su uso (limitación de la relación a/c, dosificación mínima de cemento, condiciones de curado, etc.) han dado lugar a la aparición de los problemas específicos de este cemento (conversión, hidrólisis alcalina) o la presencia, con matices propios, de fenómenos comunes a otros tipos de hormigones (reducción de la alcalinidad de la pasta cementante).

Estos fenómenos de naturaleza química llevan asociadas consecuencias tales como la disminución significativa de resistencias, o la corrosión de armaduras (en presencia de humedad) (Fig. 1), que pueden afectar de forma grave a la función mecánico-resistente para la que las viguetas habían sido fabricadas.

La constatación de esta situación, ha hecho necesario el abordar, con mayor o menor intensidad, campañas de auscultación de los edificios en cuya construcción fue presumiblemente utilizado el cemento aluminoso.

Este artículo tiene por objeto comentar las líneas generales de tal tipo de auscultaciones, presentando las posibilidades de aplicación al caso, de la técnica específica de la medida de la intensidad de corrosión de las armaduras, así como algunos resultados estadísticos obtenidos a partir de reconocimientos efectuados por el Laboratorio GEOCISA.

\section{ACTUACIÓN EN EDIFICIOS CONSTRUIDOS CON CEMENTO ALUMINOSO}

\subsection{Detección de la presencia de cemento aluminoso}

Resulta obvio que el paso previo necesario ante la sospecha de que la estructura de un edificio pueda estar afectada por la problemática propia de los elementos fabricados con cemento aluminoso es la confirmación de la presencia de este tipo de conglomerante.

Diferentes técnicas de análisis químico (determinación de óxidos de aluminio, hierro y calcio mediante espectrometría de plasma u otras técnicas, test de oxina y sulfatos, etc.) han sido aplicadas con éxito permitiendo resolver la inmensa mayoría de los casos sin necesidad de recurrir a métodos más sofisticados.

Debe subrayarse, sin embargo, la conveniencia de que este primer paso parta de la observación visual de un cierto número de viguetas que permita detectar el posible uso en el edificio, o conjunto de edificios, de diferentes tipos de viguetas. En todo caso, cabe recurrir a tomar muestras y realizar análisis químicos preferentemente en las viguetas que por su aspecto hagan sospechar con más fundamento la presencia de cemento aluminoso, con el objeto de limitar en lo posible el coste de esta etapa de identificación del tipo de conglomerante y detección de la presencia de cemento aluminoso.

\subsection{Evaluación de la degradación sufrida}

\subsubsection{Reconocimiento visual}

Una vez detectada la presencia del cemento aluminoso en un edificio o conjunto de edificios, se hace absolutamente necesario acometer un estudio más detallado que proporcione datos de cara a la adopción de decisiones tales como las de reparación o refuerzo de aquellas zonas que puedan requerirlo, o la monitorización y seguimiento de aquellas otras que se mantengan en situación aceptable.

Un análisis de esta naturaleza parte de nuevo de un reconocimiento visual de los forjados, con una intensidad considerablemente mayor que la necesaria en la fase previa de detección de la presencia de cemento aluminoso. 
Además esta intensidad no debe ser uniforme en todo el edificio sino que debe suponer un examen de casi la totalidad de las zonas húmedas, mientras en las zonas secas puede resultar considerablemente más ligera, pues aunque algunos de los fenómenos químicos antes citados pueden tener lugar sin necesidad de la presencia de un cierto grado de humedad, la existencia de ésta es determinante en el caso particular de la corrosión de las armaduras.

Este reconocimiento visual permite la detección de daños o deterioros apreciables a simple vista (fisuras, aspecto de la vigueta, roturas, desprendimientos de material, manchas de óxido, etc.) y debe complementarse con la evaluación del estado de corrosión de las armaduras.

\subsubsection{Evaluación del estado de corrosión de las armaduras}

La evaluación de la situación de corrosión de las viguetas derivada de las observaciones realizadas en el examen visual del aspecto externo, debe completarse con mediciones de tipo electroquímico. Dado que estas medidas requieren establecer contacto eléctrico con las armaduras en un punto, puede observarse la presencia de productos de oxidación, el espesor, distribución y morfología de la capa de óxidos, su coloración y la existencia o no de reducciones significativas de la sección transversal de las armaduras.

Para esta evaluación del estado de corrosión de las armaduras GEOCISA ha venido utilizando el corrosímetro portátil GECOR que permite obtener, de forma no destructiva y rápida, la velocidad instantánea de corrosión (1), (2), (3) y (4).

El método se basa en la técnica de la Resistencia de Polarización (Rp), desarrollada inicialmente para ensayos de laboratorio. Esta técnica consiste en aplicar una señal eléctrica de pequeña amplitud, obteniendo la Resistencia de Polarización mediante el cociente entre la variación del potencial y la intensidad, estando dicha Resistencia de Polarización directamente relacionada con la velocidad o intensidad de corrosión de las armaduras (Icorr).

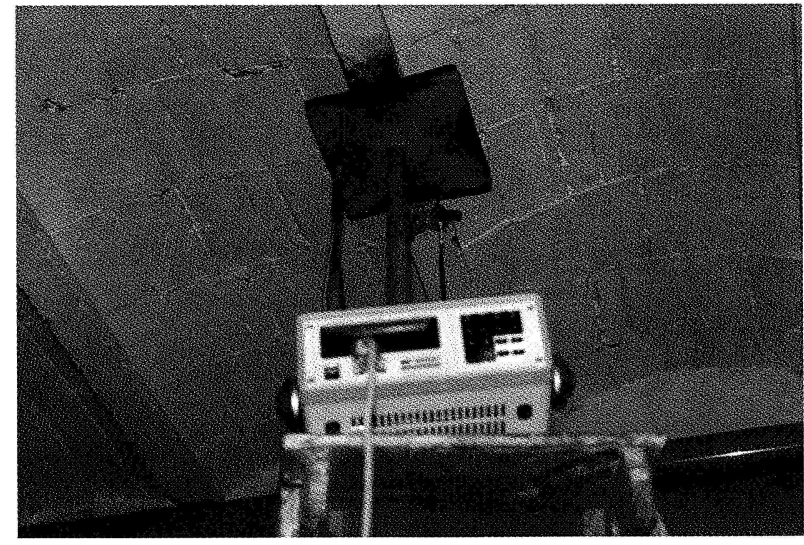

Fig. 2.-Corrosímetro "GECOR" midiendo en un forjado.

Esta técnica se aplica a estructuras de hormigón mediante el corrosímetro GECOR (Fig. 2) que utiliza el método del confinamiento sensorizado de la señal para delimitar la zona de armaduras afectadas por la polarización aplicada (5), (6).

Este corrosímetro ha sido desarrollado dentro de un proyecto EUREKA llevado a cabo en colaboración con el Consejo Superior de Investigaciones Científicas (CSIC), a través de sus institutos ICCET y CENIM, con la ayuda en su financiación del CDTI y de la Dirección General de Electrónica y Nuevas Tecnologías del Ministerio de Industria y Energía.

El citado aparato resultó muy favorablemente evaluado por el Strategic Highway Research Program (SHRP) en EE.UU. frente a otros sistemas para la medida de velocidades de corrosión desarrollados en el propio EE.UU. y Japón (7).

El corrosímetro proporciona el valor de la velocidad o intensidad de corrosión en $\mu \mathrm{A} / \mathrm{cm}^{2}$. Velocidades de corrosión inferiores a $0,1 \mu \mathrm{A} / \mathrm{cm}^{2}$ indican que el acero está pasivo, y corresponden a $2,2 \times 10^{-3} \mathrm{~mm}$ de disminución de diámetro al año, en los supuestos de corrosión homogénea y de que se mantenga constante la velocidad de corrosión medida. Por el contrario, velocidades comprendidas entre 1 y $10 \mu \mathrm{A} / \mathrm{cm}^{2}$ corresponden a situaciones de corrosión importantes y suponen pérdidas de diámetro al año comprendidas entre $2,2 \times 10^{-2}$ y $2,2 \times 10^{-1} \mathrm{~mm}$, en los mismos supuestos anteriores (Fig. 3). 


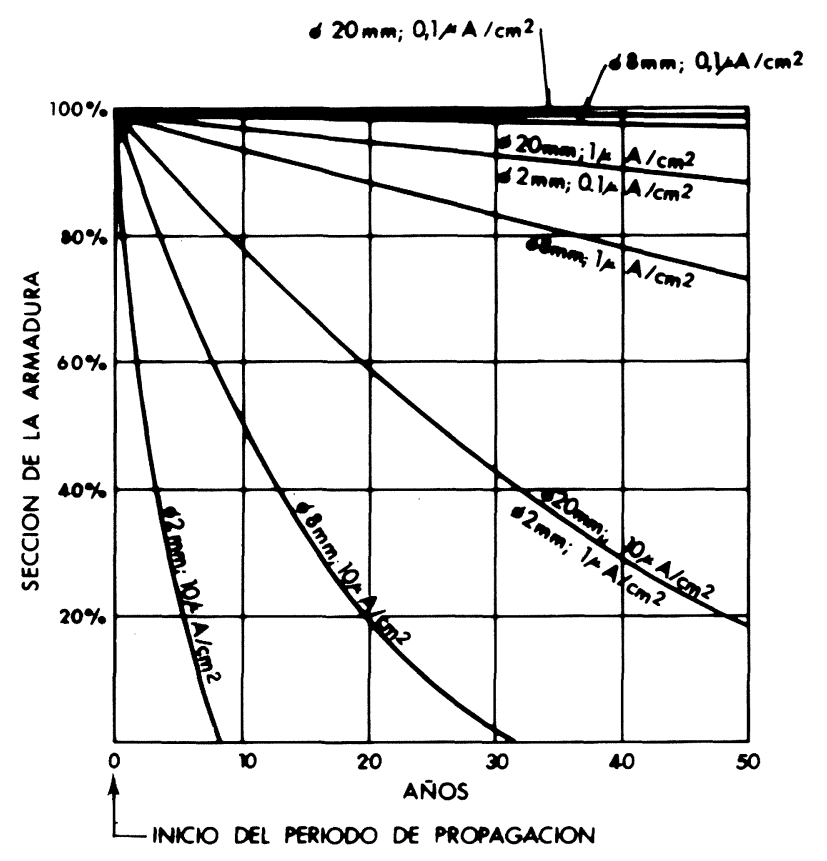

Fig. 3.-Disminución de la sección de la armadura con el tiempo, para distintas velocidades de corrosión.

El corrosímetro proporciona también la medida del potencial electroquímico de corrosión de las armaduras embebidas en el hormigón (8). Esta medida indica, de forma no cuantitativa y aproximada, la situación de corrosión o pasividad de las armaduras. En principio, potenciales comprendidos entre +100 y -200 milivoltios (E.C.S.) son sinónimo de bajo riesgo de corrosión mientras que potenciales más negativos que -350 milivoltios indican un alto riesgo de corrosión. Resulta, por tanto, una medida cualitativa que puede resultar complementaria de la medida de Icorr, aunque no sustitutiva de esta última (9), ya que:

- la medida de intensidades de corrosión no sólo confirma la presencia o no de procesos activos de corrosión sino que proporciona una cuantificación de los mismos,

- y permite tal confirmación y cuantificación incluso en los casos correspondientes al intervalo de valores del potencial en que la presencia de fenómenos activos de corrosión es dudosa.

Finalmente, el corrosímetro proporciona también la medida de la resistividad del hormigón, como parámetro que, al estar relacionado con la humedad contenida en sus poros, resulta importante conocer para una adecuada interpretación de las medidas de Icorr realizadas.
Las medidas con el corrosímetro requieren la comprobación de la sección de las armaduras para cuantificar la superficie lateral afectada por la señal eléctrica aplicada. En el caso de viguetas se realiza una pequeña roza transversal al eje de las viguetas que permite determinar el número de armaduras de refuerzo existentes en la capa inferior y si están o no conectados eléctricamente entre sí.

\subsubsection{Evaluación de la degradación del hormigón.}

Finalizado el reconocimiento visual se hace necesario acometer un estudio de la degradación del hormigón, desde el punto de vista químico, en las distintas zonas del edificio.

Su objetivo es determinar el tipo de degradación y realizar una evaluación cualitativa del estado actual de la misma, y su posible evolución.

En las zonas con daños aparentemente graves es conveniente este examen con vistas a confirmar el tipo y grado de alteración química que los ha ocasionado, aunque lógicamente debe acometerse su refuerzo en cualquier caso, por lo que su intensidad puede ser inferior.

En las restantes zonas (sin daños o con daños leves), debe también estudiarse la situación del hormigón de cemento aluminoso desde un punto de vista químico para conocer el tipo y grado de alteración alcanzado, y los riesgos potenciales que, por consiguiente, presenta ese hormigón, de sufrir nuevas evoluciones que empeoren su situación respecto a la del momento del examen.

La etapa de reconocimiento visual y evaluación de la corrosión permite la clasificación de las distintas zonas del edificio, según ese criterio de daños aparentes, facilitando la selección de los puntos donde efectuar la extracción de muestras para la realización de ensayos.

Para esos estudios de la degradación del hormigón se emplean diferentes ensayos químicos (determinación del $\mathrm{pH}$, determinación del conglomerante utilizado, análisis termogravimétrico, difracción de Rayos $\mathrm{X}$, porosidad, etc.) que no sólo aportan información complementaria a la evaluación de la situación de corrosión de armaduras antes comentada (caso de la determinación del pH) 
sino que permiten analizar si se han producido degradaciones, y el tipo de alteración preponderante.

La realización previa de los ensayos de determinación del tipo de conglomerante en las distintas zonas permitiría acotar, en su caso, la posible existencia de zonas en las que el cemento empleado no sea aluminoso, en cuyo caso podría descartarse la realización de ensayos relativamente costosos (difracción de Rayos X, análisis termogravimétrico), replanteando el enfoque de las actuaciones a adoptar en esas zonas según presenten o no daños aparentes más o menos importantes.

\subsubsection{Evaluación resistente del forjado}

Como ya se comentó en párrafos anteriores, si bien la naturaleza de los fenómenos de degradación que se presentan en los hormigones fabricados con cemento aluminoso es de tipo químico, tiene consecuencias directas importantes sobre el comportamiento mecánico resistente del elemento estructural estudiado.

Desgraciadamente, no existe, hoy por hoy, posibilidad alguna de relacionar cuantitativamente el tipo y grado de alteración química con la pérdida de resistencias mecánicas.

Paralelamente, tampoco puede establecerse una relación cuantitativa directa entre la situación de corrosión de las armaduras y la pérdida de capacidad portante del elemento estructural afectado, salvo adoptando hipótesis aproximadas no suficientemente verificadas experimentalmente (10). En este campo se están iniciando esfuerzos de investigación prometedores como el proyecto BRITE de investigación actualmente en desarrollo, en el que colaboran instituciones y empresas españolas (CSIC-ICCET, GEOCISA), británicas (BCA) y suecas (Lund Institute, CBI, CEMENTA). Uno de los objetivos de este proyecto es estudiar cómo afecta la corrosión de las armaduras a la fisuración del hormigón, la adherencia de las armaduras y, en último término, a la capacidad portante del elemento afectado.

Por todo ello, si se quieren adoptar decisiones de mantenimiento de elementos sin daños o con daños aparentemente leves, fundamentadas en datos que fueran algo más que estimaciones cualitativas de niveles de daños, parece necesario acometer una determinación de las características resistentes mediante ensayos directos del material, o al menos asegurar que en el momento de realizarse el estudio, la capacidad resistente es suficiente para asegurar un correcto comportamiento en servicio con un margen adecuado de seguridad.

Dada la imposibilidad práctica en el caso de los forjados, de estimar la resistencia a compresión mediante testigos extraidos con sonda a rotación, parece que el camino a seguir debe enfocarse más en la línea del ensayo directo del comportamiento resistente del forjado.

En este sentido debe recordarse que la realización de pruebas de carga "normales" (alcanzando una carga máxima no superior a la de servicio) no es suficiente cuando se trata de aceptar o rechazar un forjado no evaluable analíticamente, sino que es necesario recurrir a pruebas de cargas especiales tales como las descritas en (11) y (12).

En ambos casos se plantean pruebas en las que la carga a aplicar es superior a la máxima prevista en servicio y para la cual pretende aceptarse la estructura, estableciéndose además, criterios específicos en cuanto a deformabilidad y recuperación de flechas.

Este tipo de pruebas están recomendadas básicamente para la evaluación de estructuras trabajando fundamentalmente a flexión. En este caso, la posible disminución drástica de resistencia del hormigón puede comprometer en gran medida la respuesta de la estructura frente a E.L.U. de solicitaciones tangenciales. Aun así, se considera que tales pruebas resultarían aplicables en tanto en cuanto la aplicación de cargas máximas superiores a las previstas en la situación de servicio sin que se produzcan indicios de fallo en la estructura, supondría la garantía de un cierto nivel de seguridad frente a un posible colapso por cortante o rasante. Es evidente que, en todo caso, y tal como se indica en los dos documentos citados, las pruebas deberán realizarse adoptando una serie de medidas de seguridad para prever la situación de una posible rotura frágil por cortante o rasante.

Debe indicarse que obviamente la aceptación de forjados con este criterio basado en pruebas de carga exige la realización de un cierto número de ellas en función de la superficie total de forjados cuestionada, y que en ningún caso deberán aceptarse a través de pruebas de carga forjados de zonas en las que se hayan apreciado daños graves, las cuales deben ser reforzadas directamente. 


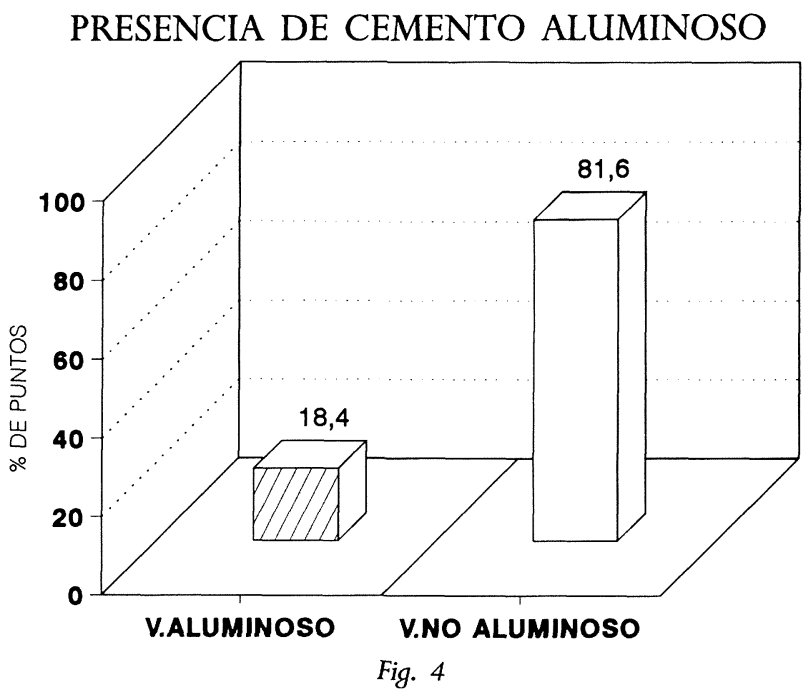

DISTRIBUCIÓN DE Icorr

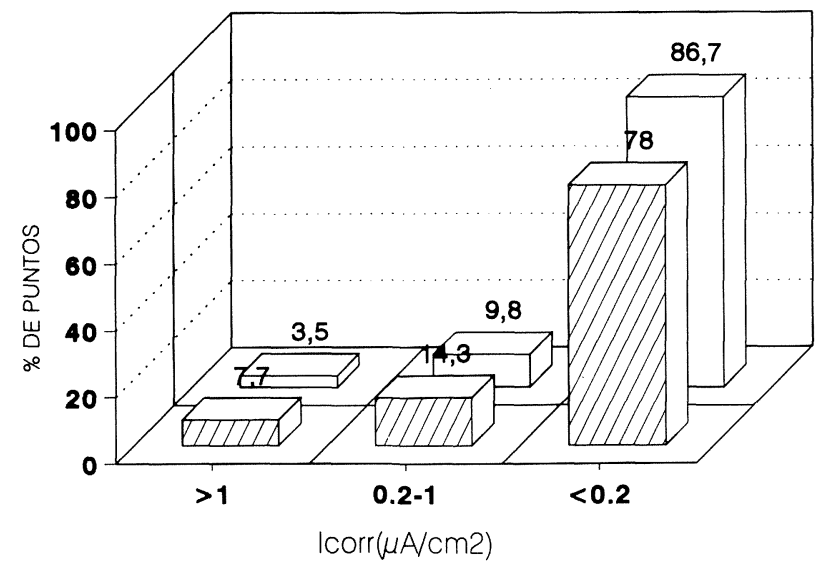

$\square$ V.ALUMINOSAS $\square$ V.NO ALUMINOSAS

Fig. 5

\section{ESTADO DE CARBONATACIÓN DEL HORMIGÓN EN CONTACTO CON LAS ARMADURAS}

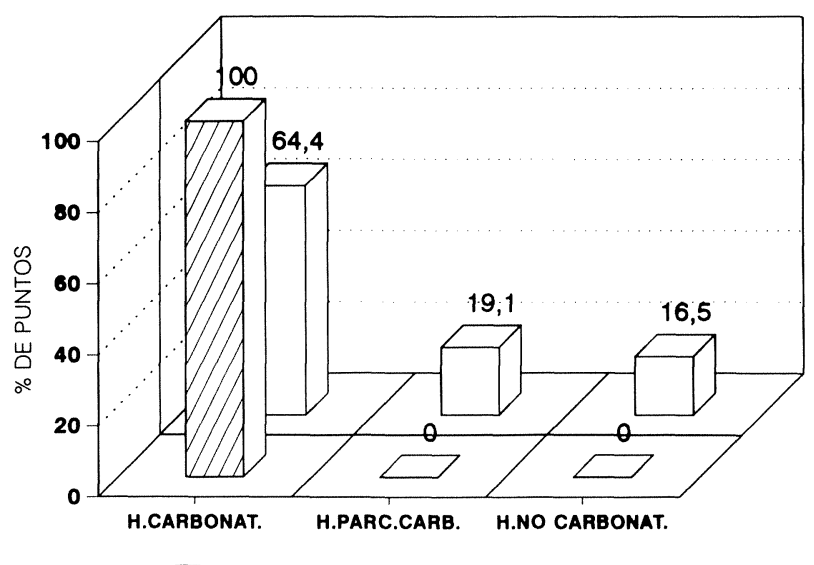

$\square$ V.ALUMINOSO $\square$ V.NO ALUMINOSO

Fig. 6
En el caso de forjados con viguetas autorresistentes, cabe la posibilidad de sustituir las pruebas de carga in situ por ensayos en laboratorio, extrayendo viguetas completas que permitirían tanto el ensayo a flexión hasta rotura de las viguetas, como el ensayo a compresión de probetas talladas a partir de las mismas.

Una auscultación del tipo de la comentada permite finalmente la definición de zonas necesitadas de refuerzo y aquellas otras en las que los forjados, en el momento de realizar la auscultación, están en situación aceptable para cumplir su función resistente. Sin embargo, en el caso del cemento aluminoso, el que esa situación sea aceptable en un momento dado, no implica que lo vaya a seguir siendo en el futuro, bien sea por la posibilidad de avance de trasnformaciones del hormigón, bien por el desarrollo de fenómenos de corrosión de las armaduras. Resulta, por tanto, obligado establecer un plan de seguimiento periódico que permita asegurar en el futuro que cualquier cambio significativo desfavorable del estado actual es detectado a tiempo de adoptar las soluciones pertinentes.

\section{RESULTADOS ESTADÍSTICOS OBTENIDOS EN LA AUSCULTACIÓN DE VIGUETAS}

En esta línea comentada, el Laboratorio GEOCISA ha venido efectuando reconocimientos de forjados presuntamente construidos con cemento aluminoso, básicamente enfocados a la detección de la presencia de este último, si bien el reconocimiento visual y ensayos químicos de identificación del conglomerante se han complementado con el estudio de la situación de corrosión de las armaduras y realización de medidas de la velocidad de corrosión de las mismas.

Los datos estadísticos que se presentan están realizados sobre una población de 1000 viguetas que fueron objeto de este tipo de auscultación.

A partir de los datos recogidos, las viguetas auscultadas fueron clasificadas cualitativamente en cuatro niveles según su situación de deterioro, correspondiendo el 1 al máximo nivel de daños y el 4 al mínimo. En esa clasificación se han tenido en cuenta no sólo los daños observados en el reconocimiento visual, sino el tipo de conglomerante, y el estudio de corrosión de armaduras. 
De estos 1000 puntos auscultados, en un $18,4 \%$ se ha detectado presencia de cemento aluminoso (Fig. 4).

La intensidad de corrosión de las armaduras registrada en cada uno de los puntos refleja una cierta influencia (Fig. 5), del tipo de conglomerante empleado, de forma que, en el caso de viguetas fabricadas con cemento aluminoso, la proporción de casos con velocidades elevadas o intermedias es mayor que en ausencia de este tipo de cemento.

En línea con este resultado, el tipo de cemento empleado también resulta fundamental en el proceso de carbonatación del hormigón, de forma que en todas las viguetas fabricadas con cemento aluminoso, el hormigón en contacto con las armaduras se encuentra totalmente carbonatado (Fig. 6). Sin embargo, en las viguetas no aluminosas, aunque en más de la mitad de los casos el hormigón se encuentra totalmente carbonatado, existen algunos casos de carbonatación parcial (sólo en algunos puntos del contacto hormigón-armaduras), e incluso armaduras en contacto con hormigón que aún mantiene su carácter básico pasivante.

El efecto del tipo de cemento empleado se manifiesta de forma aún más significativa en los niveles de daños con los que han sido calificados los puntos auscultados, que como ya se ha comentado son resultado de todos los indicadores obtenidos en la auscultación, entre los que se encuentran la intensidad de corrosión medida, y el estado de la vigueta. Debe significarse que la idea de asociar el concepto de nivel de daño con el de mayor o menor periodicidad en la reinspección de la vigueta, determinó que, aún en el caso de ausencia de daños aparentes, el simple hecho de la presencia de cemento aluminoso llevase aparejado la clasificación en el nivel 3 y no en el 4. Este criterio justifica la no presencia de viguetas aluminosas clasificadas en el nivel 4, pero afectaría en muy escasa medida al hecho significativo de los considerablemente mayores porcentajes de viguetas clasificadas en los niveles 1 y 2 dentro de la población de las fabricadas con cemento aluminoso (Fig. 7).

Del total de viguetas auscultadas, casi la mitad son pretensadas, correspondiendo dos terceras partes de la otra mitad a viguetas armadas y el resto a viguetas cerámicas u otros tipos (Fig. 8).

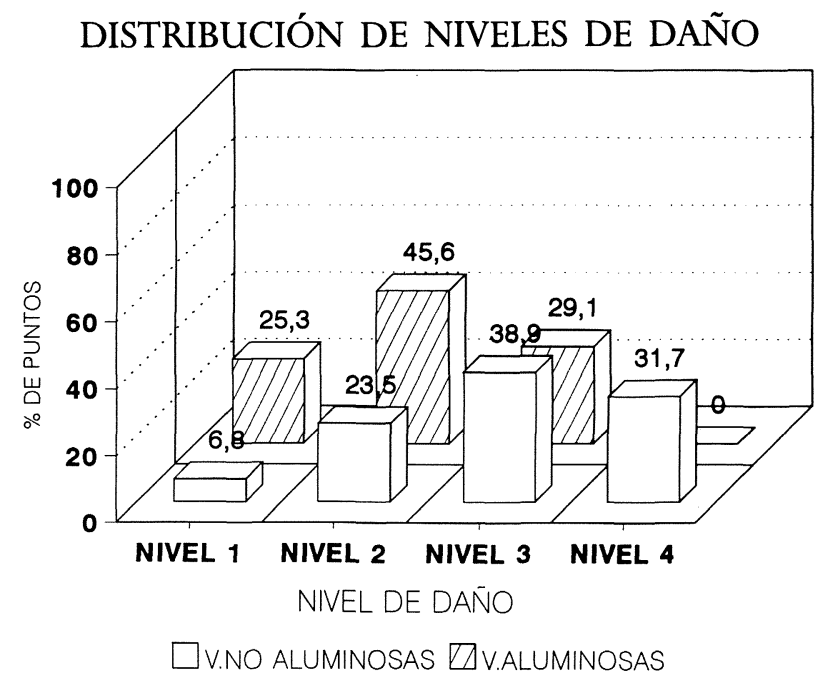

Fig. 7

\section{TIPOLOGÍA DE VIGUETAS}

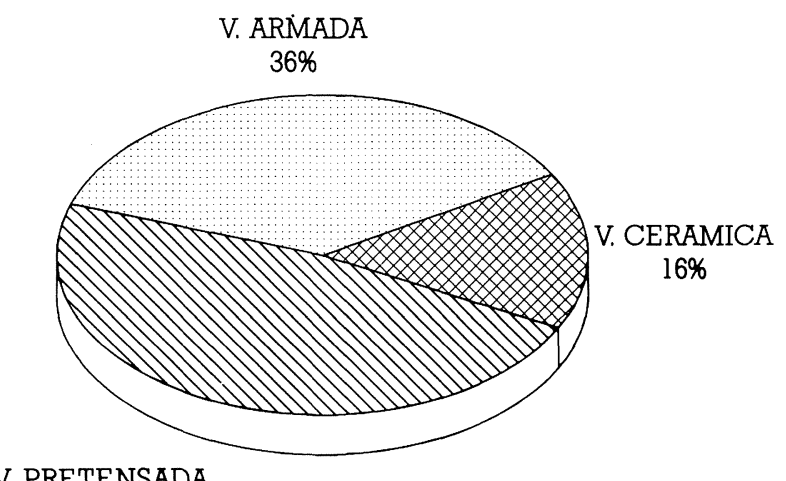

V. PRETENSADA

$48 \%$

Fig. 8
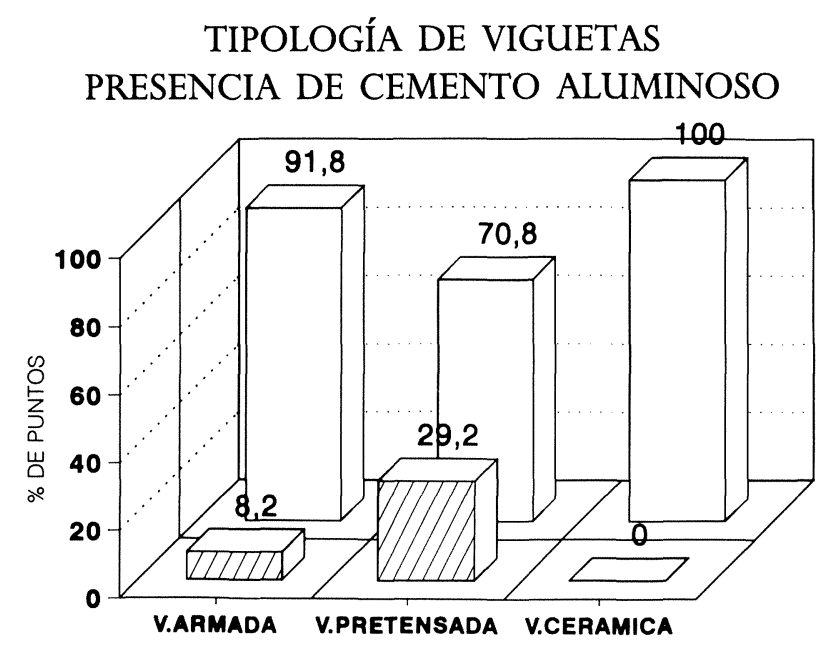

$\square$ C.ALUMINOSO $\square$ C. NO ALUMINOSO

Fig. 9 


\section{TIPOLOGÍA DE VIGUETAS DISTRIBUCIÓN DE Icorr}

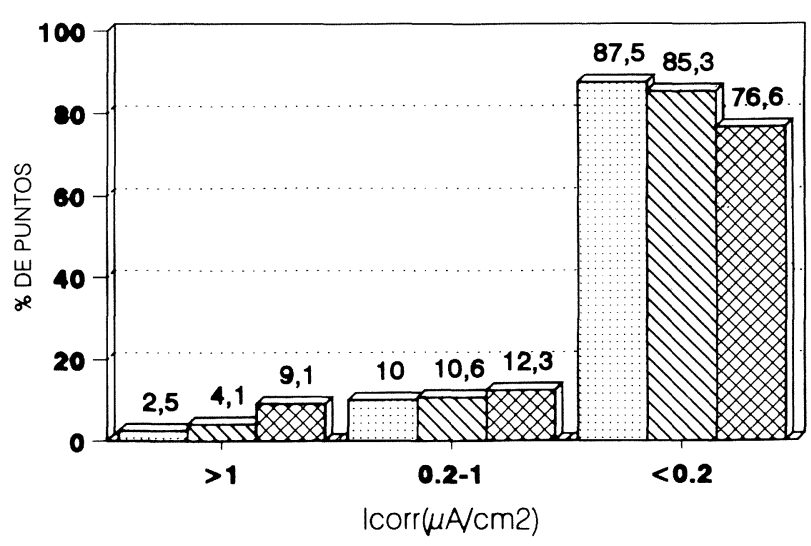

V.ARMADAS $\mathbb{Q}$ V.PRETENSADAS $\otimes$ V.CERAMICAS

Fig. 10

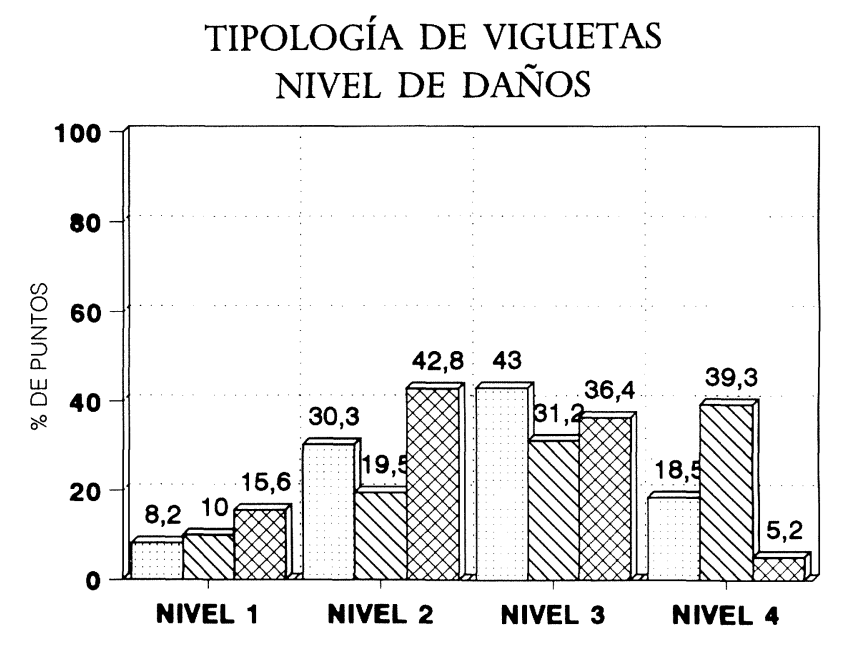

VV.ARMADAS $\$$ V.PRETENSADAS $\otimes$ V.CERAMICAS

Fig. 11
La mayoría de las viguetas fabricadas con cemento aluminoso son pretensadas, aunque se ha detectado también en viguetas armadas. La figura 9 refleja la incidencia de la presencia de cemento aluminoso detectada en los distintos tipos de viguetas. Como puede verse, el porcentaje de viguetas con cemento aluminoso es más de tres veces superior entre las viguetas pretensadas, llegando a ser casi un tercio del total de viguetas pretensadas auscultadas.
El análisis de la distribución de los valores de Icorr en función de la tipología de viguetas (Fig. 10) indica una tendencia clara a que se presenten mayores intensidades de corrosión en las viguetas cerámicas pese a no estar construidas con cemento aluminoso, posiblemente por tratarse de elementos con un nivel de ejecución poco cuidadoso.

En este mismo sentido, la figura 11 muestra la distribución de niveles de daño en función de la tipología de viguetas, pudiéndose observar que nuevamente son las viguetas cerámicas, a pesar de no estar nunca fabricadas con cemento aluminoso, las que presentan un nivel de daños más acusado.

\section{CONSIDERACIONES FINALES}

Se presentan en este artículo algunos aspectos de la auscultación de forjados construidos con cemento aluminoso, comentando las líneas generales de actuación en los edificios cuando se sospeche la presencia de cemento aluminoso en los mismos y la aplicación que puede tener al caso la medida de la velocidad de corrosión de las armaduras.

Se indican asimismo algunos resultados estadísticos de auscultaciones llevadas a cabo sobre más de 1000 viguetas en los que se pone de manifiesto la tendencia a la presencia de daños más graves en viguetas construidas con cemento aluminoso, sin que en forma alguna pueda olvidarse el menor, pero considerable, porcentaje de casos con daños aparentes graves detectados en forjados que no utilizaban ese tipo de conglomerante.

En los casos auscultados, la práctica totalidad de los daños graves detectados están asociados a importantes procesos de corrosión, ya sean activos actualmente o en el pasado. Este hecho subraya el interés que, en la auscultación, puede tener el estudio de la situación de corrosión de las armaduras, para detectar tales procesos activos de corrosión antes de que den lugar a deterioros apreciables. En este sentido conviene resaltar que en casi un $40 \%$ de los casos en los que la intensidad de corrosión registrada era mayor que $0,5 \mu \mathrm{A} / \mathrm{cm}^{2}$, no se detectaban todavía daños aparentes ocasionados por esa corrosión. 
Por otra parte, no debe olvidarse que los procesos patológicos que se presentan en el hormigón de cemento aluminoso pueden afectar de forma muy significativa a su resistencia, por lo que, a priori, no puede garantizarse que la ausencia de daños visibles en general, y de situaciones de corrosión de armaduras en particular, sea sinónimo de una capacidad portante con un nivel de seguridad adecuado.
En este sentido sería de gran interés que, según vayan acumulándose datos de la situación resistente real de casos estudiados, éstos vayan publicándose, de forma que puedan enfocarse las futuras actuaciones de auscultación de la manera más eficaz posible.

\section{BIBLIOGRAFÍA}

(1) C. Andrade, S. Feliú, J. A. González, C. Alonso, A. M. García: "On-site corrosion rate measurement in chloride contaminated concrete structures". Presented at ACI Spring Convention, Boston, USA, March 1991.

(2) J. Rodríguez: "Aplicación de la medida de la velocidad de corrosión de las armaduras al estudio de estructuras de hormigón". I Simposio Nacional sobre Conservación, Rehabilitación y Gestión de Puentes, pp. 211-220. Asociación Técnica de Carreteras (Comité Español de la AIPCR), Madrid, Junio 1991.

(3) J. Rodríguez, L. M. Ortega, A. M. García: “Contribución al estudio de las estructuras de hormigón de edificación mediante la medida de la velocidad de corrosión de sus armaduras". Congreso Internacional sobre Rehabilitación del Patrimonio Arquitectónico y Edificación, pp. 362-365. Tenerife, Julio 1992.

(4) C. Andrade, C. Alonso, L. M. Ortega, A. M. García: "On-site monitoring of concrete repairs by polarization resistance". Proceedings of International RILEM/CSIRO/ACRA Conference on Rehabilitation of Concrete Structures, pp. 51-60, Melbourne, Australia, September 1992.

(5) S. Feliú, J. A. González, S. Feliú Jr., C. Andrade: "Confinement of the electrical signal for in-situ measurement of Polarization Resistance in reinforced concrete". ACI Materials Journal, September - October 1990, pp. 457-460.

(6) V. Ausín, J. Rodríguez, L. M. Ortega: "Measurement of corrosion rates on RC structures: A contribution to the assessment of damaged structures". Bulletin nr. 106 of the IASS, pp. 87-93.
(7) SHRP: Condition Evaluation of concrete bridges relative to reinforcement corrosion. Vol. 2: "Method for measuring the corrosion of reinforcing steel". Strategic Highway Research Program, National Research Council, SHRP-S/FR-92-104, Washington 1992.

(8) ASTM C876: "Standard test method for half-cell potentials of uncoated reinforcing steel in concrete".

(9) S. Feliú, C. Andrade: "Manual de inspección de obras dañadas por corrosión de armaduras". Programa temático "Corrosión y protección de materiales". Subprograma "Corrosión de armaduras". I.E. Torroja (CSIC). 1989.

(10) J. Rodríguez, C. Andrade: "Influencia de la corrosión de las armaduras en la capacidad portante de las estructuras de hormigón armado". Hormigón y Acero n.o 175, pp. 119-124, 1990.

(11) Grupo Español del Hormigón (GEHO): "Evaluación de la Capacidad Portante de Estructuras mediante pruebas de carga". Boletín n. 1 del GEHO. Comisión IV - Grupo de Trabajo IV-3. Madrid, Diciembre 1988.

(12) ACI 437R-91 (Revisión 1982): "Strength evaluation of existing concrete buildings". Comité ACI 437, Detroit, 1992. 